\title{
THE HOMOGENEOUS APPROXIMATION PROPERTY AND THE COMPARISON THEOREM FOR COHERENT FRAMES
}

\author{
KARLHEINZ GRÖCHENIG
}

\begin{abstract}
We show that the homogeneous approximation property and the comparison theorem hold for arbitrary coherent frames. This observation answers some questions about the density of frames that are not covered by the theory of Balan, Casazza, Heil, and Landau. The proofs are a variation of the method developed by Ramanathan and Steger.
\end{abstract}

Frames provide redundant non-orthogonal expansions in Hilbert space, intuitively they should therefore be "denser" than orthonormal bases. The first fundamental result is Landau's necessary condition for sets of sampling of band-limited functions [11]. A second fundamental idea is the homogeneous approximation property and the comparison theorem of Ramanathan and Steger [12] in the context of Gabor frames. Since then many contributions have investigated the density of frames and varied and applied the method of Ramanathan and Steger. See $[3,4,8,13]$ for a sample of papers. The approach in [12] culminates in the deep investigation of Balan, Casazza, Heil, and Landau $[1,2]$ who have found a density theory for a general class of frames that are labeled by a discrete Abelian group.

However, the BCHL theory is not universally applicable, for instance, the density of wavelet frames does not fall under this general theory, and a separate investigation is necessary, as was shown in a sequence of papers by Heil and Kutyniok $[9,10]$ and by Sun [14-16]. Their work uses quite explicit and long calculations in the group of affine transformations.

In this note we show that the approach of Ramanathan and Steger yields the homogeneous approximation property and a comparison theorem for the class of coherent frames. These frames arise as subsets of the orbit of a square-integrable group representation. In particular, wavelet frames possess this structure. The advantage of the abstract point of view is the conciseness and simplicity of the proofs and the much more general range of validity. The additional insight is that the homogeneous approximation property and the comparison theorem are a consequence of structure alone, we do not need to assume additional localization properties as in [1].

We will work in the context of a locally compact group $G$ with Haar measure $d x$. We write $|U|=\int_{G} \chi_{U}(x) d x$ for the measure of a set $U \subseteq G$ and $K^{c}$ for the complement of $K$ in $G$.

Key words and phrases. Frame, square-integrable representation, homogeneous approximation property, comparison theorem, density.

K. G. was supported by the Marie-Curie Excellence Grant MEXT-CT 2004-517154. 
Amalgam spaces. Let $U=U^{-1} \subseteq G$ be a symmetric compact neighborhood of the identity element $e \in G$ and let

$$
f^{\sharp}(x)=\sup _{y \in x U}|f(y)|
$$

be the local maximum function of $f$. Then the left amalgam space $W\left(L^{\infty}, L^{2}\right)$ is defined by the norm

$$
\|f\|_{W\left(L^{\infty}, L^{2}\right)}=\left\|f^{\sharp}\right\|_{2} .
$$

Recall that a set $\mathcal{X} \subseteq G$ is called relatively (left) separated if

$$
\sup _{x \in G} \operatorname{card}(\mathcal{X} \cap x U)<\infty \text {. }
$$

for some, hence all, compact neighborhood(s) $U$ of $e$. Equivalently, for any compact set $K \subseteq G$ the sum $\sum_{x \in \mathcal{X}} \chi_{x K}$ is uniformly bounded in $G$.

Lemma 1. Let $\mathcal{X}=\left\{x_{j}\right\}$ be a relatively (left) separated set in $G$ and $K \subseteq G$ a compact set. Then

$$
\sum_{x_{j} \notin K}\left|f\left(x_{j}\right)\right|^{2} \leq C \int_{K^{c} U}\left|f^{\sharp}(x)\right|^{2} d x .
$$

Proof. Set $C_{0}=\left\|\sum_{j \in J} \chi_{x_{j} U}\right\|_{\infty}$. By (2) this constant is finite. First we note that $\left|f\left(x_{j}\right)\right| \leq f^{\sharp}(x)$ whenever $x_{j} \in x U$ or $x \in x_{j} U$ (because $U$ is symmetric). Consequently

$$
\left|f\left(x_{j}\right)\right|^{2} \leq \frac{1}{|U|} \int_{x_{j} U} f^{\sharp}(x)^{2} d x .
$$

So after summing over $j$, we obtain

$$
\begin{aligned}
\sum_{x_{j} \notin K}\left|f\left(x_{j}\right)\right|^{2} & \leq \frac{1}{|U|} \sum_{x_{j} \notin K} \int_{x_{j} U} f^{\sharp}(x)^{2} d x \\
& \leq \frac{1}{|U|} C_{0} \int_{K^{c} U} f^{\sharp}(x)^{2} d x,
\end{aligned}
$$

because $\sum_{x_{j} \notin K} \chi_{x_{j} U}(x) \leq C_{0} \chi_{K^{c} U}(x)$ for all $x \in G$.

Next we consider the setup for coorbit theory and coherent frames. Let $(\pi, \mathcal{H})$ be a unitary, square-integrable representation of $G$ on a Hilbert space $\mathcal{H}$. For fixed $g \in \mathcal{H}, g \neq 0$, we set $V_{g} f=\langle f, \pi(x) g\rangle$; this representation coefficient is the analog of the wavelet transform or short-time Fourier transform for the group $G$.

We investigate frames of the form $\left\{\pi\left(x_{j}\right) g: j \in J\right\}$, so-called coherent frames. This means that there exist positive constants $A, B>0$, the frame bounds, such that

$$
A\|f\|^{2} \leq \sum_{j}\left|\left\langle f, \pi\left(x_{j}\right) g\right\rangle\right|^{2}=\sum_{j}\left|V_{g} f\left(x_{j}\right)\right|^{2} \leq B\|f\|^{2}
$$

for all $f \in \mathcal{H}$. From (4) it is clear that the sampling of the representation coefficients with respect to $g$ on the set $\mathcal{X}$ must be in $\ell^{2}$. This is close, but not equivalent, to 
saying the $V_{g} f \in W\left(L^{\infty}, L^{2}\right)$. Thus the frame condition motivates the following definition.

Definition 1. A vector (wavelet) $g \in \mathcal{H}$ is "nice", if the mapping $V_{g}$ (the generalized wavelet transform) maps $\mathcal{H}$ into $W\left(L^{\infty}, L^{2}\right)$.

In most situations the set of nice vectors is dense in $\mathcal{H}$ and examples can be easily constructed (see Remark 1). In the following we assume that $\left\{\pi\left(x_{j}\right) g: j \in J\right\}$ is a frame for $\mathcal{H}$ and that $\left\{h_{j}: j \in J\right\}$ is a dual frame. We may take any dual frame, it need not be the canonical dual. We remark that $\left\{x_{j}\right\}$ must be relatively separated in this case (otherwise the upper frame bound would not exist).

The intuition behind coherent frames is that the frame vector $\pi\left(x_{j}\right) g$ lives near the point $x_{j}$ in the "G-plane". Consequently, a vector of the form $\sum_{x_{j} \in K} c_{j} \pi\left(x_{j}\right) g$ should live on $K$.

To formalize this intuition, we introduce the following subspaces of $\mathcal{H}$. Let $L \subseteq G$ be a compact subset of $G$ and $y \in G$, then $V_{L, y}$ is the subspace of $\mathcal{H}$ spanned by the vectors of the dual frame $h_{j}, x_{j} \in y L$. Formally,

$$
V(y L)=\operatorname{span}\left\{h_{j}: x_{j} \in y L\right\},
$$

and we let $P_{L, y}$ be the orthogonal projection from $\mathcal{H}$ onto $V(y L)$.

The following homogeneous approximation property is a weak form of localization of a frame. The proof for coherent frames follows closely the proof for bandlimited functions in [8].

Proposition 2 (Homogeneous Approximation Property). Assume that $g$ is nice and that $\left\{\pi\left(x_{j}\right) g: j \in J\right\}$ is a frame for $\mathcal{H}$ and that $\left\{h_{j}: j \in J\right\}$ is a dual frame. Then for every $f \in \mathcal{H}$ and $\epsilon>0$ there exists a compact set $L \subseteq G$ (depending on $f$ and $\epsilon$ ) such that

$$
\sup _{x \in y K}\left\|\pi(x) f-P_{K L, y} \pi(x) f\right\|<\epsilon
$$

holds for all compact sets $K \subseteq G$ and all $y \in G$.

Proof. Since $g$ is nice, the representations coefficient $V_{g} f$ is in $W\left(L^{\infty}, L^{2}\right)$ for all $f \in L^{2}$. Therefore for any $\delta>0$ there is a compact set $L \subseteq G$, such that on $L^{c} U$, an open neighborhood of the complement of $L$, the maximal function $\left(V_{g} f\right)^{\sharp}$ is small, precisely,

$$
\int_{L^{c} U}\left(V_{g} f\right)^{\sharp}(x) d x<\delta .
$$

We will use the frame expansion

$$
\pi(x) f=\sum_{j}\left\langle\pi(x) f, \pi\left(x_{j}\right) g\right\rangle h_{j} .
$$

Recall the following property of orthogonal projections: If $P_{K L, y} h=\sum_{j: x_{j} \in y K L} c_{j} h_{j}$, then

$$
\left\|h-P_{K L, y} y\right\| \leq\left\|h-\sum_{j: x_{j} \in y K L} d_{j} h_{j}\right\|
$$


for any choice of coefficients $d_{j}$. Choosing the special coefficients of the frame expansion (8), we obtain that

$$
\begin{aligned}
\left\|\pi(x) f-P_{K L, y} \pi(x) f\right\|^{2} & \leq\left\|\pi(x) f-\sum_{j: x_{j} \in y K L}\left\langle\pi(x) f, \pi\left(x_{j}\right) g\right\rangle h_{j}\right\|^{2} \\
& =\left\|\sum_{j: x_{j} \notin y K L}\left\langle\pi(x) f, \pi\left(x_{j}\right) g\right\rangle h_{j}\right\|^{2} \\
& \leq A^{-1} \sum_{j: x_{j} \notin y K L}\left|\left\langle\pi(x) f, \pi\left(x_{j}\right) g\right\rangle\right|^{2} \\
& =A^{-1} \sum_{j: x_{j} \notin y K L}\left|\left\langle f, \pi\left(x^{-1} x_{j}\right) g\right\rangle\right|^{2} .
\end{aligned}
$$

The last inequality follows from the fact that $\left\{h_{j}\right\}$ is a frame and obeys the Bessel inequality with constant $A^{-1}$, where $A$ is the lower frame bound of $\left\{\pi\left(x_{j}\right) g\right\}$ in (41).

Some gymnastics: Since $x_{j} \notin y K L$, we have $x^{-1} x_{j} \notin x^{-1} y K L$. Further, $\left\{x^{-1} x_{j}\right\}$ is relatively separated, and therefore

$$
\left\|\sum_{j} \chi_{x^{-1} x_{j} U}\right\|_{\infty}=C_{0}<\infty
$$

where this constant is independent of $x$.

We apply Lemma 1 and obtain

$$
\begin{aligned}
\sum_{j: x^{-1} x_{j} \notin x^{-1} y K L}\left|\left\langle f, \pi\left(x^{-1} x_{j}\right) g\right\rangle\right|^{2} & =\sum_{j: x^{-1} x_{j} \notin x^{-1} y K L}\left|V_{g} f\left(x^{-1} x_{j}\right)\right|^{2} \\
& \leq C \int_{\left(x^{-1} y K L\right)^{c} U}\left|V_{g} f^{\sharp}(x)\right|^{2} d x
\end{aligned}
$$

So for $K \subseteq G$ compact and $x \in y K$ we obtain

$$
\left\|\pi(x) f-P_{K L, y} \pi(x) f\right\|^{2} \leq A^{-1} C \sup _{x^{-1} y=z \in K^{-1}} \int_{(z K L)^{c} U} V_{g} f^{\sharp}(x)^{2} d x .
$$

If $K \subseteq G$ is compact and $x \in y K$, then $z=x^{-1} y \in K^{-1}$, therefore $L \subseteq z K L$ for all $z \in K^{-1}$ and $(z K L)^{c} U \subseteq L^{c} U$. Therefore

$$
\sup _{z \in K^{-1}} \int_{(z K L)^{c} U} V_{g} f^{\sharp}(u)^{2} d u \leq \int_{L^{c} U}\left(V_{g} f\right)^{\sharp}(u)^{2} d u .
$$

If we now choose $L$ such that $C A^{-1} \int_{L^{c} U}\left(V_{g} f\right)^{\sharp}(u)^{2} d u<\epsilon$, which is possible by (77), then we have proved that

$$
\sup _{x \in y K}\left\|\pi(x) f-P_{K L, y} \pi(x) f\right\|<\epsilon
$$

and the homogeneous approximation property is proved.

REMARK: In most situations, nice vectors $g$ exist in abundance. For instance, if $(\pi, \mathcal{H})$ is irreducible and square-integrable, then a nice vector can be constructed as follows: Let $g_{0} \in \mathcal{H}$ be admissible, and $k$ be continuous with compact support. 
Then $g=\pi(k) g_{0}=\int k(x) \pi(x) g_{0} d x$ has the property that $V_{g} f^{\sharp} \in W\left(C, L^{2}\right)$ for all $f \in \mathcal{H}$. This follows from a convolution equation in [5]. See also [6].

If $\pi$ is the reducible representation by translations and dilations on $L^{2}\left(\mathbb{R}^{d}\right)$ defined by $\pi(x, s) f(t)=\left|\operatorname{det} e^{-s A / 2}\right| f\left(e^{-s A}(t-x)\right.$, where $x, t \in \mathbb{R}^{d}, s \in \mathbb{R}^{+}$and $A$ is a real-valued $d \times d$-matrix with all eigenvalues having positive real part, then every Schwartz function $g$ such that $\operatorname{supp} \hat{g} \subseteq\{\omega: 0<a \leq|\omega| \leq b\}$ is nice. This follows immediately from the fact that the wavelet transform $V_{g} g(x, s)=\langle g, \pi(x, s) g\rangle$ has compact support in $s$. See [7] for more on admissible vectors in this case.

The Comparison Lemma. Before we estimate the dimension of the finitedimensional subspaces defined in (5), we state a simple estimate for the trace of an operator.

Lemma 3. Let $T$ be a positive trace-class operator on a Hilbert space $\mathcal{H}$ and $\left\{h_{k}\right.$ : $k \in J\}$ be a frame with frame bounds $A, B>0$. Then

$$
\frac{1}{B} \sum_{k}\left\langle T h_{k}, h_{k}\right\rangle \leq \operatorname{tr} T \leq \frac{1}{A} \sum_{k}\left\langle T h_{k}, h_{k}\right\rangle .
$$

Proof. By the spectral theorem there exists an orthonormal set $\varphi_{j}$ and positive eigenvalues $\lambda_{j}$ such that $T f=\sum_{j} \lambda_{j}\left\langle f, \varphi_{j}\right\rangle \varphi_{j}$. Then

$$
\begin{aligned}
\sum_{k}\left\langle T h_{k}, h_{k}\right\rangle & =\sum_{j} \sum_{k} \lambda_{j}\left|\left\langle\varphi_{j}, h_{k}\right\rangle\right|^{2} \\
& \leq B \sum_{j} \lambda_{j}\left\|\varphi_{j}\right\|_{2} \\
& =B \sum_{j} \lambda_{j}=B \operatorname{tr} T
\end{aligned}
$$

Likewise, by using the lower frame bound, we obtain $\sum_{k}\left\langle T h_{k}, h_{k}\right\rangle \geq A \operatorname{tr} T$.

Let $\mathcal{E}_{g}=\left\{\pi\left(x_{j}\right) g: x_{j} \in \mathcal{X}\right\}$ and $\mathcal{E}_{h}=\left\{\pi\left(y_{k}\right) h: y_{k} \in \mathcal{Y}\right\}$ be two coherent frames. As above, denote the dual frame of $\mathcal{E}_{g}$ by $\left\{h_{j}\right\}$ and its frame bounds by $A, B$.

For compact subsets $K, L \subseteq G$ and $y \in G$, define the subspaces

$$
\begin{aligned}
V_{g}(y K L) & =\operatorname{span}\left\{h_{j}: x_{j} \in y K L\right\} \\
W_{h}(y K) & =\operatorname{span}\left\{\pi\left(y_{j}\right) h: y_{k} \in y K\right\},
\end{aligned}
$$

and let $P=P_{g}(y K L)$ and $Q=Q_{h}(y K)$ be the orthogonal projections onto the subspaces $V_{g}$ and $W_{h}$. Finally let $T=Q P Q: W_{h} \rightarrow W_{h}$. T is a positive operator of finite rank. We estimate its trace from above and below.

To keep track of these subspaces and projections, keep in mind that $\mathcal{E}_{g}$ is a given frame whose density we want to understand and that $\mathcal{E}_{h}$ is the reference frame. If possible, $\mathcal{E}_{h}$ is chosen to be a Riesz basis or an orthonormal basis. This has been the case in most applications of the comparison theorem so far. 
Theorem 4. Given $\epsilon>0$, there exists a compact set $L \subseteq G$, such that for all $y \in G$ and all compact sets $K \subseteq G$

$$
\|h\|^{2} B^{-1}(1-\epsilon) \operatorname{card}\left\{k: y_{k} \in y K\right\} \leq \operatorname{card}\left\{x_{j} \in y K L\right\} .
$$

Proof. Since $T$ is a product of orthogonal projections, its eigenvalues are between 0 and 1 and

$$
\operatorname{tr} T \leq \operatorname{rank} P \leq \operatorname{card}\left\{x_{j} \in y K L\right\} .
$$

To obtain a lower bound, we use Lemma 3 and obtain

$$
\begin{aligned}
\operatorname{tr} T & \geq \frac{1}{B} \sum_{k}\left\langle T \pi\left(y_{k}\right) h, \pi\left(y_{k}\right) h\right\rangle \\
& \geq \frac{1}{B} \sum_{k: y_{k} \in y K}\left\langle T \pi\left(y_{k}\right) h, \pi\left(y_{k}\right) h\right\rangle \\
& =\sum_{k: y_{k} \in y K}\left\langle Q P Q \pi\left(y_{k}\right) h, \pi\left(y_{k}\right) h\right\rangle \\
& =\frac{1}{B} \sum_{k: y_{k} \in y K}\left\langle P \pi\left(y_{k}\right) h, \pi\left(y_{k}\right) h\right\rangle \\
& =\frac{1}{B} \sum_{k: y_{k} \in y K}\left(\left\langle\pi\left(y_{k}\right) h, \pi\left(y_{k}\right) h\right\rangle+\left\langle(\mathrm{I}-P) \pi\left(y_{k}\right) h, \pi\left(y_{k}\right) h\right\rangle\right. \\
& =\frac{\|h\|^{2}}{B} \operatorname{card}\left\{k: y_{k} \in y K\right\}+(*) .
\end{aligned}
$$

To estimate $(*)$, we apply the homogeneous approximation property (Proposition 2). Choose a compact set $L \subseteq G$ such that

$$
\left\|\pi(x) h-P_{K L, y} \pi(x) h\right\|<\epsilon\|h\|
$$

for all compact sets $K \subseteq G$ and $x \in y K$. Then

$$
\begin{aligned}
(*) & \leq \frac{1}{B} \sum_{k: y_{k} \in y K}\left\|\pi\left(y_{k}\right) h-P\left(\pi\left(y_{k}\right) h\right)\right\|\left\|\pi\left(y_{k}\right) h\right\| \\
& \leq \frac{1}{B} \sum_{k: y_{k} \in y K} \epsilon\|h\|\left\|\pi\left(y_{k}\right) h\right\|=\frac{1}{B} \operatorname{card}\left\{k: y_{k} \in y K\right\} \epsilon\|h\|^{2} .
\end{aligned}
$$

By combining these estimates we obtain a lower bound

$$
\sum_{k: y_{k} \in y K}\left\langle T \pi\left(y_{k}\right) h, \pi\left(y_{k}\right) h\right\rangle \geq \operatorname{card}\left\{k: y_{k} \in y K\right\}\|h\|^{2} B^{-1}(1-\epsilon) .
$$

Combining (14) and (15), the claim is proved.

The comparison theorem is the starting point for genuine density theorems. For instance, Landau's necessary conditions for set of sampling are a simple corollary of Theorem 4. Likewise, Ramanathan and Steger derived the necessary density 
of nonuniform Gabor frames as an immediate corollary of a special case of Theorem 4. For more general groups and representations, one may define some form of a Beurling density and deduce a density theorem, but the results are not completely satisfying.

\section{REFERENCES}

[1] R. Balan, P. G. Casazza, C. Heil, and Z. Landau. Density, overcompleteness, and localization of frames. I. Theory. J. Fourier Anal. Appl., 12(2):105-143, 2006.

[2] R. Balan, P. G. Casazza, C. Heil, and Z. Landau. Density, overcompleteness, and localization of frames. II. Gabor systems. J. Fourier Anal. Appl., 12(3):309-344, 2006.

[3] O. Christensen, B. Deng, and C. Heil. Density of Gabor frames. Appl. Comput. Harmon. Anal., 7:292-304, 1999.

[4] H. Feichtinger, M. Neuhauser, and M. Piotrowski. The homogeneous approximation property for coorbit spaces. In preparation.

[5] H. G. Feichtinger and K. Gröchenig. Banach spaces related to integrable group representations and their atomic decompositions. I. J. Functional Anal., 86(2):307-340, 1989.

[6] H. Führ and K. Gröchenig. Sampling theorems on locally compact groups from oscillation estimates. Math. Z., 255(1):177-194, 2007.

[7] K. Gröchenig, E. Kaniuth, and K. F. Taylor. Compact open sets in duals and projections in $L^{1}$-algebras of certain semi-direct product groups. Math. Proc. Cambridge Philos. Soc., 111(3):545-556, 1992.

[8] K. Gröchenig and H. Razafinjatovo. On Landau's necessary density conditions for sampling and interpolation of band-limited functions. J. London Math. Soc. (2), 54(3):557-565, 1996.

[9] C. Heil and G. Kutyniok. Density of weighted wavelet frames. J. Geom. Anal., 13(3):479-493, 2003.

[10] C. Heil and G. Kutyniok. The homogeneous approximation property for wavelet frames. $J$. Approx. Theory, 147:28-46, 2007.

[11] H. J. Landau. Necessary density conditions for sampling and interpolation of certain entire functions. Acta Math., 117:37-52, 1967.

[12] J. Ramanathan and T. Steger. Incompleteness of sparse coherent states. Appl. Comput. Harmon. Anal., 2(2):148-153, 1995.

[13] A. P. Schuster. The homogeneous approximation property in the Bergman space. Houston J. Math., 24(4):707-722, 1998.

[14] W. Sun. Density of wavelet frames. Appl. Comput. Harmon. Anal., 22(2):264-272, 2007.

[15] W. Sun. Homogeneous approximation property for wavelet frames. Preprint, 2007.

[16] W. Sun and X. Zhou. Density of irregular wavelet frames. Proc. Amer. Math. Soc., 132(8):2377-2387 (electronic), 2004.

Faculty of Mathematics, University of Vienna, Nordbergstrasse 15, A-1090 ViENNA, AUSTRIA

E-mail address: karlheinz.groechenig@univie.ac.at 